\title{
ERRATUM TO: ON SOME PROBLEMS OF EUCLIDEAN RAMSEY THEORY
}

\author{
I. D. SHKREDOV \\ Steklov Mathematical Institute of RAS, Gubkina str. 8, 119991 Moscow, Russia \\ e-mail: ilya.shkredov@gmail.com
}

Erratum to: Analysis Mathematica 41 (4) (2015) 299-310

DOI: $10.1007 / \mathrm{s} 10476-015-0304-1$

The following Acknowledgement was inadvertently omitted from my above article:

This work was supported by grant Russian Scientific Foundation RSF 14-11-00433.

The online version of the original article can be found under DOI: 10.1007/s10476-015-0304-1. 